\title{
A Framework with OTSU'S Thresholding Method for Fruits and Vegetables Image Segmentation
}

\author{
Mukesh Kumar Tripathi \\ Research Scholar, Department of CSE \\ GNDEC, Bidar, Karnataka, India
}

\author{
Dhananjay D. Maktedar, PhD \\ Professor, Department of CSE \\ GNDEC, Bidar, Karnataka, India
}

\begin{abstract}
An accurate technique for segmentation of fruits and vegetables image is vital and major challenges in computer vision. Various segmentation techniques are available in digital image processing. In this paper, we introduce a framework for fruits and vegetables background subtraction employing Otsu's algorithm. This method is widely used in various image segmentation applications. The Otsu's method is useful in subtraction of background under the partial effect of occlusion, cropping, noisy and blurred images. Our proposed method was experimented by employing fruit and vegetable images acquired locally. Our experimental results confirm that, Otsu's threshold based method is able to extract fruit and vegetable objects with good accuracy.
\end{abstract}

\section{Keywords}

Otsu's method, Image segmentation, Fruits and vegetables Image, Morphological Operation, Thresholding Method

\section{INTRODUCTION}

We live in a digital world where digital Image plays a crucial role of conveying information through different medium [1]. Image processing has been widely used in assessing the quality of agriculture products. The agriculture based image processing methods are found to suffer from noisy image acquisition. This noise is contributed mainly from unbalance illumination, external noise, image occlusion and other possible reason. In order to reduce the effect of noise in acquired agriculture based image it is essential to perform background extraction operation. The extracted image after removal of noise enhances computational efficiency and accuracy of results. Image segmentation methods are also helpful in enhancing the quality of Images. This has several applications in medical field, Image retrieval, face recognition, Biometrics, Video Surveillance, Object detection, military, remote sensing and agriculture etc.

Segmentation is quite useful in various applications $[2,3$, 4,5]. Many researchers are working on segmentation techniques, especially in medical and agricultural fields, but still it is a 'grand challenge' in the computer vision for all researchers. The agriculture field plays a crucial role in an Indian economy. Near about 70 percentages population of India depends on agriculture [6].

We know that the value of fruits and vegetables directly proposal to quality of product. The assessment of quality of fruits and vegetables can be done by traditional method but it is very time consuming and not able to produce accurate results. Due to availability of the supermarket we required more efficient and accurate system for background extraction.

Image sets of pixels, each pixel having some attribute. In the segmentation techniques all are grouped with similar attribute pixel. That's why segmentation is an important and challenging task in image processing. For example, suppose we have an image with collection of object and we want to identify and classify one particular object so we need to apply segmentation method to extract the background of the image.

Similarity and detection of discontinuity play an important role in segmentation of an image in the form of point detection, line detection and edge detection. Segmentation method can be broadly classified into six categories.

Histogram Based Image Segmentation methods can be used to find out the appropriate value of the thresholding [7]. The histogram of image calculated by the number of pixels having a same gray level later perform the normalization operation on pixel represents the value between 0 and 1 . This technique is very effective to enhance with contrast of images. One disadvantage of this method is that it could not be much more effective for color images. One other disadvantage of this method is that it appeared in the different range base of gray value so discontinuity may be possible in the segmentation.

Edge Based Image Segmentation a method is broadly used techniques when it measures with other segmentation methods. It relies on modelling of edge intuitively. Pewit and Sobel mask use gradient operator to detect the edge with include isotropic results. The one main disadvantage of this method is that it does not able to perform admirably well when edges are blurred and noisy [8].

Region Based Image Segmentation methods is quite useful when the region is massive. The main objective of this method to split and merge the regions.

Hephzibah A. Christian et al., [9] uses a Region Based Image Segmentation approach for 2D digital image. Many researchers frequently used automatic region based algorithms for remote sensing application [10]. A sometime fusion of region based and edge based method provide effective results [11]. The disadvantage of this method is that it is sensitive to noise computationally expensive.

A neural Network Based Image Segmentation method relies on the hidden layer. This hidden layer act as a bridge between input and output. In this, for desired output weight is adjusted by neurons in each layer. The disadvantage of this method is that it generates only rules and results produced by the neural network are quite difficult to understand.

Clustering Based Image Segmentation methods, choice of number of clusters is crucial for the final computations [12]. K-means Clustering Based Image Segmentation methods are more popular and widely used by many researchers due to its simplicity and effectiveness [13].

Clustering method is broadly classified in three categories (Supervised clustering, Semi Supervised clustering, unsupervised clustering). Unsupervised clustering one method among all others which are commonly used by researcher $[14$, $15,16]$. In medical application most researchers have used 
fuzzy, c-means (FCM) method because it produced effective results of de- noising of image [17], This FCM method classifies the region into the different cluster and obtained centroid point. Many works are proposed using Thresholding Based Image Segmentation methods by many researchers $[18,19,20]$. This paper presents an effective image segmentation approach using Otsu's Thresholding Based Image Segmentation.

Finally, with all above step we will able extract the background of fruits and vegetables. The rest paper is organized as follows: Section 2 present a brief overview of related work. Section 3 describes proposed method for background subtraction. Section 4 gives the details of the database. Section 5 demonstrates the segmentation results and last section 6 concludes with final remarks.

\section{RELATED WORK}

For Background subtraction, we have used thresholding based image segmentation method and applied on dataset various fruits and vegetables. In this section, we will concentrate on a brief overview of related work in segmentation.

Shiv ram Dubey and Anand Jalal [22, 23] have used K-Means clustering techniques for background subtraction. For the case study they have used different category of fruits and vegetables. For example, sample image size of the fruits and vegetables is reduced by 25 percentages and performed The $\mathrm{K}$-means Clustering by $\mathrm{K}=2$ values on resizing images and finally perform the morphological closing operation to close small holes.

Many authors have used K-Means clustering approach for image segmentation [24, 25]. By using K-means clustering algorithms author is able to extract background of those images having normal illumination, noise and blurred effect. In the clustering based segmentation method one problem arises, how to decide the number of clusters. Many researchers have given their view no one has been reached with hundred percentage accuracy to solve this problem. Due to this it is open and challenging task for all the researcher's accuracy to solve this problem.

Sinthia P, and K. Sujatha [26] proposed improved K-means method for background extraction in medical field. They have used first filtering techniques to remove unwanted noise they define the value of the cluster. Many researchers discuss all types of segmentation methods used in various applications $[27,28,29]$.

Segmentation is a core part core part in image processing and computer vision application such as medical image segmentation, face recognition, disease recognition, handwritten recognition, traffic control system, video surveillances etc. segmentation in medical field broadly classifies in two forms such as partial automatic [30,31] and completely automatic $[32,33]$. Chandra S, Bhat R, Singh H [34] proposed restoration and clustering based segmentation of brain tumor. This both segmentation method produced better results compared to other segmentation method in medical fields.

Hsin-Chia Chen and Sheng-Jyh Wang [35] evaluate the level of color segmentation by comparison between JSEG algorithms and mean shift algorithms. Pre-processing is also important task which mostly involved in removing of unwanted noise [36, 37]. T. Gomati and B. L. Shiva Kumar [38] also present important of pre-processing operation on an image before segmentation.
Segmentation is quite useful and essential in medical application. Ze-Xuan Ji et al. [39] used a new method with modified FCM for brain MR segmentation. In this proposed algorithm the input is brain MR, the image is represented in the histogram. All the matrix value is normalized between 0 and1. The next step involved in calculating maxima and minima of the histogram and last output is represented in the form of brain MR. Researcher also used atlas -based method for automatic segmentation of MR image [40].

Bacon Zheng, Zhang Yi [41] also proposed a CLM based method for MR image segmentation. Due to distortion MR image has unwanted noise. Using CLM method, they divide the image into sub images that are segmented individually.

Now day storing and classification of data is a challenging task due to easy availability of data through social media and other medium. Seema Wazarkar et al., [42] used region based segmentation method for background removal of social network image and classification is done using $\mathrm{KNN}$ and Soft KNN classifier which provide better results.

Jianping Fan et al., [43] proposed automatic image segmentation, color edge extraction and seed region growing. In the color edge detection, image is extracted in $\mathrm{Y}, \mathrm{U}, \mathrm{V}$ component and next obtained gradient of $\mathrm{Y}, \mathrm{U}, \mathrm{V}$ component by applying pattern based gradient calculation, later apply thresholding based edge detection method to obtain an edge of $\mathrm{Y}, \mathrm{U}, \mathrm{V}$ component individually. At last obtain results in the form of a map edge.

Another method using seed region growing was proposed based on convention region growing [44]. Frank Y. Shih, Shouxian Cheng [45] proposed color image segmentation. Initially the component in RGB color space is transformed into $\mathrm{Y}, \mathrm{Cb} \mathrm{Cr}$ color space. Seed growing and region merging techniques are used for color segmentation and Similarity measure is done by the standard derivation of $\mathrm{Y} \mathrm{Cb} \mathrm{Cr}$ component. Another color image segmentation using CLPSObased Fuzzy method is present by Mandana Hamidi and Ali Borji [46]. Many researchers have also used Fuzzy based clustering techniques for segmentation [47].

G Rajesh Chandra et al., [48] Proposed Genetic algorithms for image segmentations. Initially, it precedes the first population and calculates fitness and criteria satisfaction. If condition is satisfied, then the process is complete otherwise choosing one new population. The statistical parameter method has been used for segmentation by you. Rakesh Kumar et al., [49].

Anuradha and Bhupinder Kaur [50] use watershed segmentation method to estimate and detect the infected part of fruits. Among variance occurrence of segmentation techniques, many researchers working on unsupervised clustering. Jung-Shiong Chang et al., [51] proposed wavelet multilevel thresholding method for segmentation.

Fully automatic segmentation thresholding scheme is used by Yuan Been Chen [52]. In the initial step determines the global thresholding by part of an image frame in to $3 \times 3$ block, then find out the initial point and directions. Later divide an image frame in too many blocks and last search the contour by using modified edge schemes.

P Clarke et al., [53] present classification of segmentation into to form such as single contrast and multi spectral. Segmentation can have done by similarity measurement techniques [54]. in this they have calculated precision metric, recall Metrix and F-measure Metrix. The performance of Fmeasure metric compares effective two other two methods. 
L Deepa Parasar, Vijay R. Rathod [55] describes various segmentation methods such as Fuzzy C-mean, seed region, watershed. They have used PSO K-Clustering, Seed region PSO, Fuzzy clustering. All the results these methods are comparable in GCE index, RAND Index and VOI index. PSO $\mathrm{K}$ Clustering segmentation method produce efficient results compared to the other two methods.

Yuri Boykon et al., [56] proposed graph cut method for image object segmentation. With this method they have computer best cut and optimal cost. Fei Ni et al., [57] present DFFT method for segmentation, for case studies, they have used AR database and they have achieved 90 percentage accuracy applying this method.

\section{PROPOSED METHOD FOR BACHGROUND SUBTRACTION}

In digital image processing Image is represented by matrix form. Let us assume that image is in the RGB color model. So original image be denoted as I $r \times c \times 3$ where are represent row, c represents column and 3 channel R, G, B. As we know that each input image consists three channels in which one channel is luminance denoted by $\mathrm{Y}$ and two Chrominance channels Icb and Icr. These three Y, Icb and Icr are extracted by following equation [58].

$$
\left[\begin{array}{l}
I y \\
I C b \\
I C r
\end{array}\right]=\left[\begin{array}{l}
16 \\
128 \\
128
\end{array}\right]+\left[\begin{array}{l}
65.481 . I R+128.553 . I G+24.966 . I B \\
-37.80 . I R-74.203 . I G+112.0 .0 I B \\
112.000 . I R-93.786 . I G+18.214 . I B
\end{array}\right]
$$

One $\mathrm{Y}, \mathrm{Cb}, \mathrm{Cr}$ channel are extracted from R, G, B image, and then object is separated from background with following steps.

1. In the first step, using the Otsu's method Segmentation operation has performed on Iy channel by taking threshold value of 170. The value of threshold has been decided on the basis of the extensive image analysis method. Let the segmented image is represented by $\mathrm{D}$.

$$
D=\left[\begin{array}{lll}
0 & \boldsymbol{I} y(x, y)>170 & 0 \leq X \leq R \delta 0 \leq Y \leq C \\
1 & \boldsymbol{I} y(x, y)<170 & 0 \leq X \leq R \delta 0 \leq Y \leq C
\end{array}\right]
$$

2. After the step 1, Image has been segmented and morphological filter operation such as opening and closing method is used to remove noise from segmented image. Segmentation operation is denoted by $\mathrm{S} 1$ and morphological operation such as opening and closing method is represented such as:

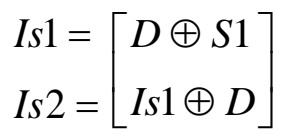

After this operation, we obtain segmented image Is which has a light background and dark object.

3. In the third step, invert the segmented image denoted by Is 1 followed by a concatenation operation to obtain an intermediate image denoted by Ib. Further extract the R, G, B channel from the intermediate Image. Last perform the concatenate the R, G, B Channel with inverted image and results is obtain as back ground subtracted image has represented by following expression This entire process is representation by (a), (b), (c).

$$
\begin{aligned}
& \left(\begin{array}{l}
\overline{I Y} \\
\overline{I C b} \\
\overline{I c r}
\end{array}\right)=\left(\begin{array}{l}
I y \\
I C b \\
I C r
\end{array}\right) \text { AND }\left(\begin{array}{l}
I s 1 \\
I s 1 \\
I s 1
\end{array}\right) \text { (a) } \\
& \left(\begin{array}{l}
\overline{I R} \\
\overline{I G} \\
\overline{I B}
\end{array}\right)=f\left(\begin{array}{l}
\overline{I y} \\
\overline{I y} \\
I y
\end{array}\right) \cdot f\left(\begin{array}{l}
\overline{I C b} \\
\overline{I C b} \\
(\underline{I C b}
\end{array}\right) \cdot f\left(\begin{array}{l}
\overline{I C r} \\
\overline{I C r} \\
\overline{\overline{I G}} \\
\overline{\overline{I B}}
\end{array}\right)=\left(\begin{array}{l}
I R \\
I G \\
I B
\end{array}\right) \text { (b) }
\end{aligned}
$$

The function "f" represent the R, G, B channel and by concatenating through modifying $\mathrm{R}, \mathrm{G}, \mathrm{B}$ channel, obtain object Ib without background. 


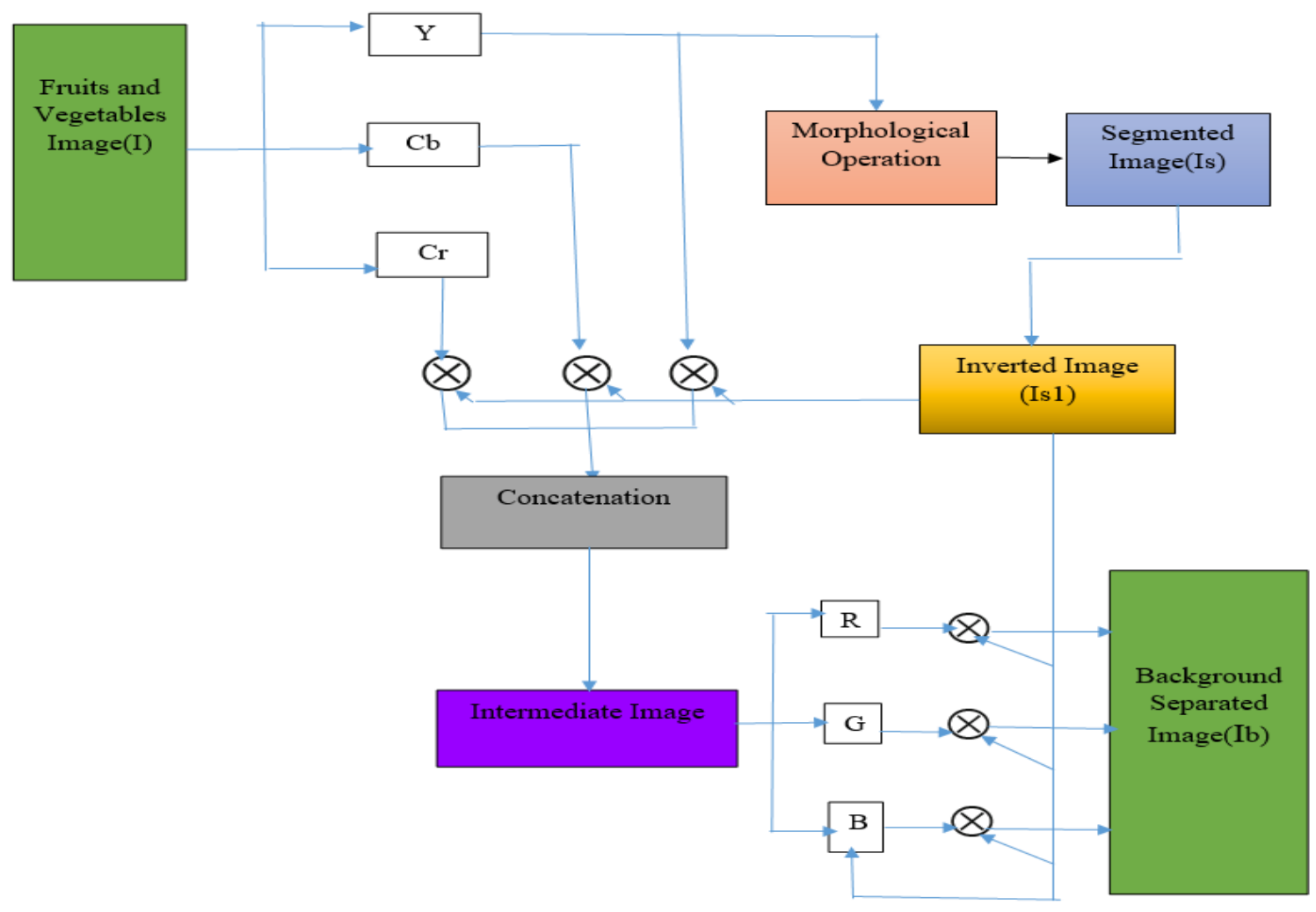

Figure 2: Block diagram of the proposed methodology

\section{RESULT AND DISUCSSION}

To evaluate the performance of proposed method, we have used a dataset with twenty different categories of fruits Sapodilla (98), Kundru (128), Sponge gourd (138), Strawberry (135), Green Tomato (180), Red Tomato (141), and Watermelon (159). For case study, we have taken 50 images from each category of fruits and vegetables.

\section{EXPERIMENT RESULTS:}

We have used Otsu's Thresholding based segmentation method for background extraction of images. Figure3. Shows the sample of image before segmentation and after segmentation. Figure 4. Shows Background subtraction results under partial occlusions and cropping effect. Figure 5. Background subtraction results under noisy and blurring effect. In the Background subtraction process, it does not separate object with in image.

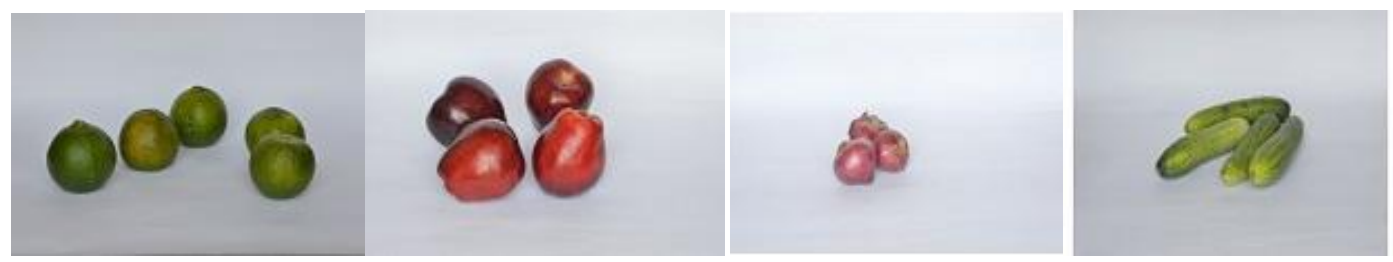

(a)

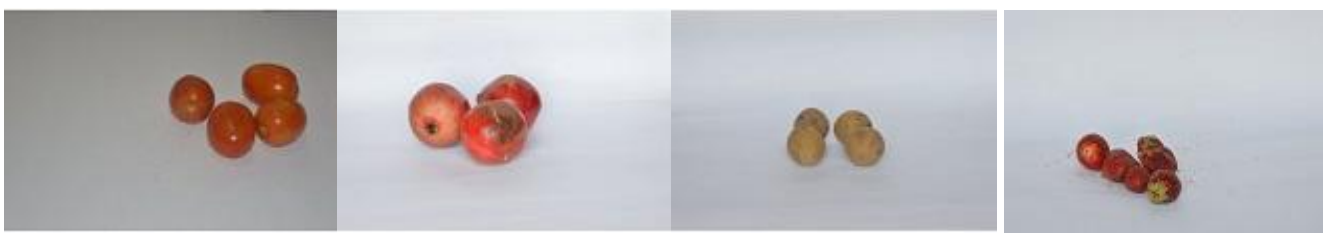

(b)

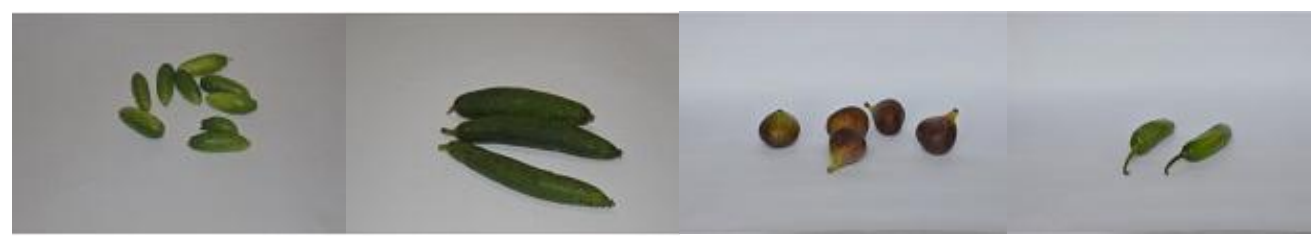

(c) 


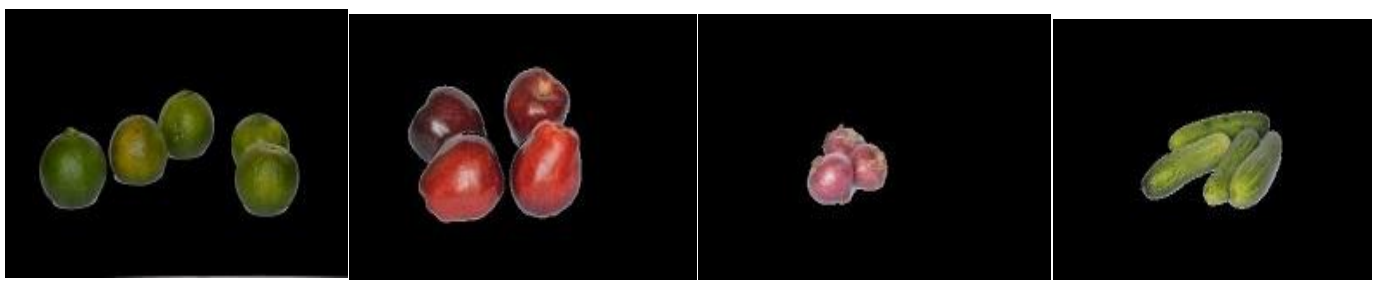

(d)

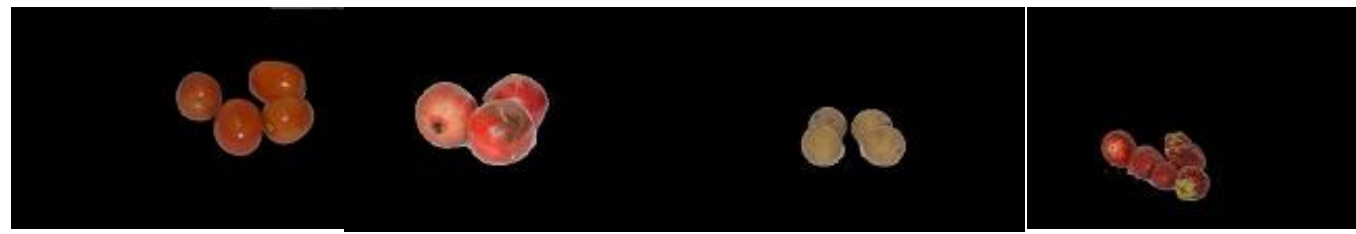

(e)

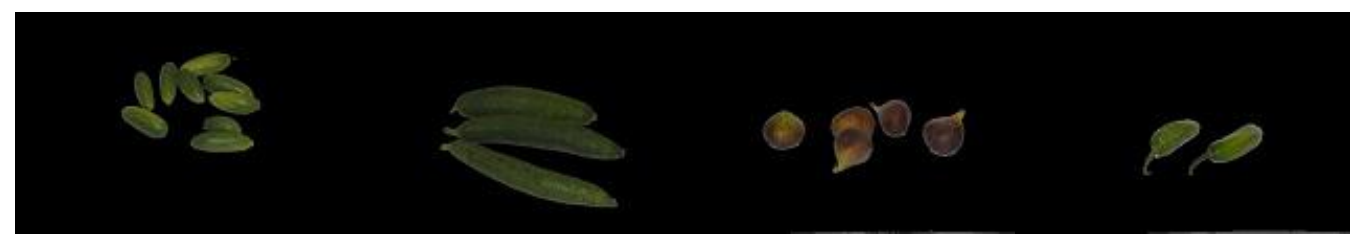

(f)

Figure 3: Extracting region of interest from the images $(a, b, c)$ before segmentation $(d, e, f)$ after segmentation.

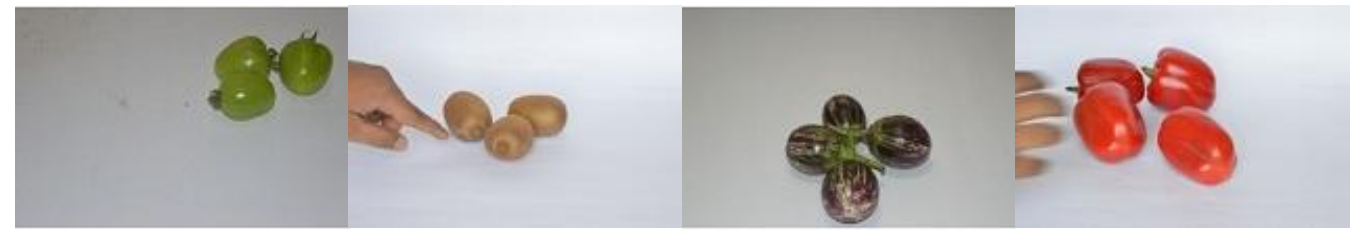

(a)

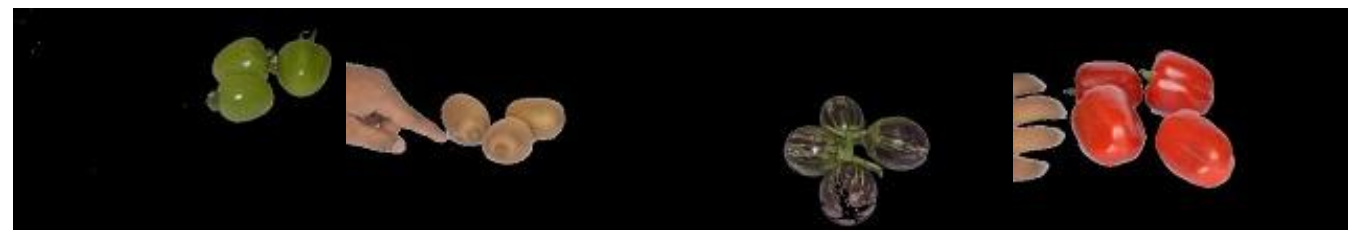

(b)

Figure 4: Background subtraction results under partial occlusions and cropping effect, (a) before segmentation, (b) after segmentation
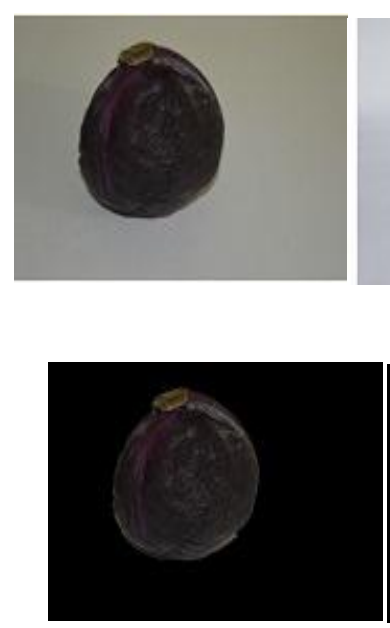
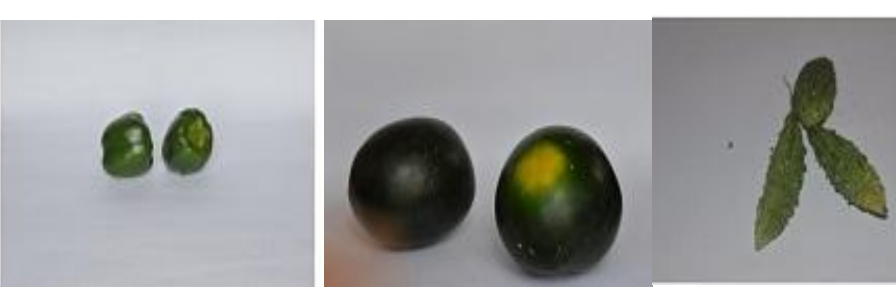

(a)
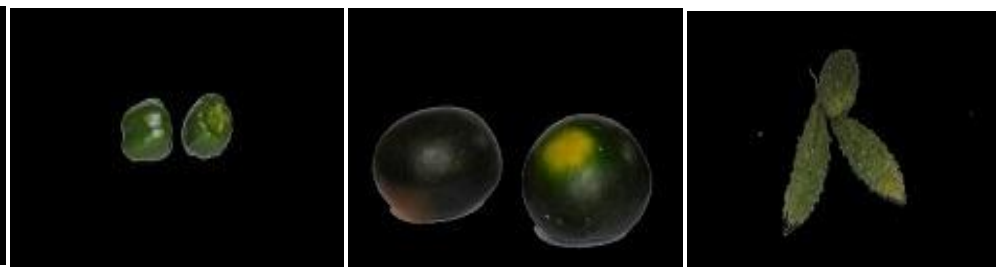

(b)

Figure 5: Background subtraction results under noisy and blurring effect, (a) before segmentation (b) after segmentation 


\section{CONCLUSION}

In this paper, we have presented the related work of all type of image segmentation method. we also proposed a framework for fruits and vegetables background subtraction which compromise in four parts: 1) Loading of image. 2) Segmentation image.3) Intermediate image.4) Background image. For background subtraction we have used Otsu's algorithms which are based on thresholding thresholding image segmentation method. This approach is evaluating on database of different category of fruits and vegetables. In the data set we have not consider the combination of different fruits and vegetables. We have taken same kind of fruits and vegetables with different pose and also variability in number. One of future direction of this work is to develop accurate recognition system of fruits and vegetables using feature extraction method based on colour and texture method and also able to classify different class of fruits and vegetables.

\section{REFERENCES}

[1] Jiawei Han, Micheline Kamber, Jian Pei. (2011).Data mining: Concepts and Techniques3rd Edition eBook ISBN: 9780123814807 . Page Count: 744.

[2] Dey, N, Roy, A, Pal, M. and Das A. (2012). Optical cup to disc ratio measurement in glaucoma diagnosis using Harris corner. Third IEEE International Conference Computing Communication and Technologies (ICCCNT12), Coimbatore.

[3] Dey, N., Roy, A., Pal, M. and Das, A. (2012). FCM based blood vessel segmentation method for retinal images. International Journal of Computer Science and Network (IJCSN). VOl. 1. NO. 3. pp.148-152.

[4]Acharjee, S. Dey, N., Biswas, D. and Chaudhuri. (2013). An efficient motion estimation algorithm using division mechanism of low and high motion zone. IEEE International Multi Conference on Automation, Computing, Control, Communication and Compressed Sensing. Kerala, India.

[5]Samanta, S., Dey, N., Das, P., Acharjee, S. and Chaudhuri, S. (2012). Multilevel threshold based grey scale image segmentation using cuckoo se arch. International Conference on Emerging Trends in Electrical, Communication and Information Technologies (ICECIT). Pp.27-34.

[6] Mukesh Kumar Tripathi, Dr. Dhananjay D. Maktedar. (2016). Recent Machine Learning Based Approaches for Disease Detection and Classification of Agricultural Products. Second IEEE International conference on computing, communication, control and automation. PCCOE, Pune.

[7] Simona Caraiman, Vasile I. Manta. (2013). Histogrambased segmentation of quantum images. Theoretical Computer Science. PP 46-60.

[8] S. Gupta, S.G. Mazumda. (2013). Sobel edge detection algorithm. Int. J. Comput. Sci.Manag. Res. Vol 2. NO.2. pp 1578-1583.

[9] Rashmi, M. Kumar, R. Saxena. (2013). Algorithm and technique on various edge detection: a survey. Signal Image Process. An Int. J. (SIPIJ). pp. 65-75.

[10] Hephzibah A. Christinal, Daniel Diaz-Pernil, Pedro Real. (2011). Region-based segmentation of 2D and 3D images with tissue-like P systems. Pattern Recognition Letters.
VOL 32, pp2206-2212.

[11] Zhongwu Wang, John R. Jensen, Jungho Im. (2010). An automatic region-based image segmentation algorithm for remote sensing applications. Environmental Modelling \& Software. VOL 20, pp 1149-1165.

[12] Kebin Wua, David Zhang. (2015). Robust tongue segmentation by fusing region-based and edge-based approaches. Expert Systems with Applications. VOL 20, pp 8027-8038.

[13] A.N. Benaichouche, H. Oulhadj, P. Siarry. (2013). Improved spatial fuzzy c means clustering for image segmentation using PSO initialization, Mahalanobis distance and post-segmentation correction. Digital Signal Processing. VOL 23, pp 1390-1400.

[14] Li-Hong Juang, Ming-Ni Wu.2010. MRI brain lesion image detection based on color-converted K-means clustering segmentation. Measurement. VOL43, pp 941949.

[15] Zhi MinWang, Yeng Chai Soh, Qing Songa, KangSim. (2009). Adaptive spatial information-theoretic clustering for image segmentation. Pattern Recognition. VOL 42, pp $2029-2044$.

[16] N.R. Pal, S.K. Pal. (1993). A review on image segmentation techniques. Pattern Recognition. pp. 1277 1294.

[17] J.C. Bezdek, L.O. Hall, L.P. Clarke. (1993). Review of MR image segmentation techniques using pattern recognition. Med. Phys. pp .1033-1048.

[18] T. Pappas. (1992). An adaptive clustering algorithm for image segmentation. IEEE Transaction Signal Process. pp. 901-914.

[19] K.P. Baby Resma, Madhu S. Nair. (2018). Multilevel thresholding for image segmentation using Krill Herd Optimization algorithm. Journal of King Saud University - Computer and Information Sciences.

[20] Sharifah Lailee Syed Abdullah, Hamirul'Aini Hambali, Nursuriati Jamil. (2012). Segmentation of Natural Images Using an Improved Thresholding-based Technique. Segmentation of Natural Images Using an Improved Thresholding-based Technique(IRIS). Procedia Engineering 41 (2012) pp 938 - 944.

[21] Xie Xie, Jiu-Lun Fan, Yin Zhub. (2011). The optimal All-Partial-Sums algorithm in commutative semigroups and its applications for image thresholding segmentation. Theoretical Computer Science. pp 1419-1433.

[22] Dubey, S. R., \& Jalal, A. S. (2013). Species and Variety Detection of Fruits and Vegetablesfrom Images. International Journal of Applied Pattern Recognition. VOL 1 .NO 1, PP $108-126$.

[23] Dubey, S. R., \& Jalal, A. S. (2015). Fruit and vegetable recognition by fusing colour and texture features of the image using machine learning. International Journal of Applied Pattern Recognition. VOL 2 NO 2, pp 160 181.

[24] Dubey, S. R., \& Jalal, A. S. (2015). Fruit and vegetable recognition by fusing colour and texture features of the image using machine learning. International Journal of Applied Pattern Recognition. VOL 2 NO 2, pp $160-$ 181. 
[25] Shiv Ram Dubey, Pushkar Dixit, Nishant Singh, Jay Prakash Gupta. Infected Fruit Part Detection using KMeans Clustering Segmentation Technique. International Journal of Artificial Intelligence and Interactive Multimedia, VOL 2, NO 2. pp. 65-72. DOI: 10.9781/ijimai.2013.229.

[26] Sinthia P, and K. Sujatha. 2016.A Novel Approach to detect bone cancer using K-means Clustering and edge detection method. ARPN Journal of Engineering and Applied Sciences. VOL. 11, NO. 13, pp 8002-8010.

[27] László Szilágyi, Sándor M. Szilágyi, Balázs Benyó, Zoltán Benyó. (2009). Application of Hybrid c-Means Clustering Models in Inhomogeneity Compensation and MR Brain Image Segmentation. Proceedings of the 7th IFAC Symposium on Modelling and Control in Biomedical Systems Aalborg, Denmark. pp 204-209.

[28] Nida M. Zaitouna, Musbah J. Aqel. (2015). Survey on Image Segmentation Techniques. International Conference on Communication, Management and Information Technology (ICCMIT). pp $797-806$.

[29] Dr. V. Seenivasagam, S. Arumugadevi.2012. A Survey of Image Segmentation Methods using Conventional and Soft Computing Techniques for Color Images. International Journal of Advanced Research in Computer Science and Electronics Engineering (IJARCSEE). VOL 1, NO 6. pp.116-121.

[30] Y.B. Chen, Oscal T.-C. Chen. (2002). Semi-automatic image segmentation using dynamic direction prediction. IEEE ICASSP. Vol 4. pp. 3369-3372.

[31] K. Karsch, Q. He, Y. Duan, A fast. (2009). semiautomatic brain structure segmentation algorithm for magnetic resonance imaging. IEEE BIBM. pp. 297-302.

[32] Y.B. Chen, O.T.-C. Chen. (2009). Image segmentation method using thresholds automatically determined from picture contents. Article ID 140492.

[33] Y.B. Chen, O.T.-C. Chen.2009. High-accuracy moving object extraction using background subtraction. ICIC Express Lett. VOL 3. pp. 933-938.

[34] Chandra S,Bhat R,Singh H.(2009). A PSO based method for detection for brain tumors from MRI. In proceding of word congress on nature and biologically insipred computing. VOL 1. pp. 666-671.

[35] Hsin-Chia Chen and Sheng-Jyh Wang. (2004). The use of visible color difference in the Quantitative evaluation of color image segmentation.

[36] Jaime S. Ide, Sheng Zhang, Chiang-shan R. Li. (2014). Bayesian network models in brain functional connectivity analysis. International Journal of Approximate Reasoning. pp.23-35.

[37] Su Ruan, Weibei Dou, Daniel Bloyet, Jean-Marc Constants. Fuzzy Fusion System for Brain MRI image Segmentation. Department of Electronic Engineering Tsinghua University. Beijing China.

[38] T. Gomathi and B. L. Shiva Kumar. (2016). A secure image encryption algorithm based ARPN Journal of Engineering and Applied Sciences. VOL. 11, NO. 1. pp 47-54.

[39] Ze-Xuan Ji, Quan-Sen Sun, De-Shen Xia. (2011). framework with modified fast FCM for brain MR images segmentation. Pattern Recognition. VOL 43. pp 9991013.

[40]M. Bach Cuadra, M. De Craene, V. Duaya, B. Macq, C. Pollo, J.-Ph. Thiran. (2006).Dense deformation field estimation for atlas-based segmentation of pathological MR brain images. computer methods and programs in biomedicine. VOL 84. pp 66-75.

[41] Bochuan Zheng, Zhang Yi. (2012). A new method based on the CLM of the LV RNN for brain MR image segmentation. Digital Signal Processing. Vol 22.pp.497505 .

[42] Seema Wazarkar, Bettahally N. Kesavmurty, Ahsan Hussain. (2017). Region based segmentation of social image using soft $\mathrm{KNN}$ algorithms $.6^{\text {th }}$ international conference on smart computing and communication (ICSCC). pp 93-98.

[43] Jianping Fan, David. K. Y. Yau, Ahmed. K. Elmagarmid, Walid G. Aref, Automatic Image Segmentation by Integrating Color-Edge Extraction and Seeded Region Growing. IEEE TRANSACTIONS ON IMAGE PROCESSING, VOL. 10, NO. 10. pp 1454-1466.

[44] Rolf Adams and Leanne Bisch of. (1994).Seeded Region Growing.IEEE Transactions on Pattern analysis and machine intelligence. VOL. 16,NO. 6, pp 641-647.

[45] Frank Y. Shih, Shouxian Cheng. (2005). Automatic seeded region growing for color image segmentation Image and Vision Computing. VOL 23.PP. 877-886.

[46] Mandana Hamidi and Ali Borji. (2007). Color Image Segmentation with CLPSO-based Fuzzy IJCSNS International Journal of Computer Science and Network Security. VOL.7 No.6. pp 215-221.

[47] Feng Zhao, Licheng Jiao, Hanqiang Liu. (2013). Kernel generalized fuzzy c-means clustering with spatial information for image segmentation. Digital Signal Processing. VOL 23, pp. 184-199.

[48] G Rajesh Chandra, Dr. Kolasani Ram Chand H Rao, (2016). Tumor detection in brain using genetic algorithm.7th International Conference on Communication, Computing and Virtualization. Procedia Computer Science 79. pp. $449-457$.

[49] Y. Rakesh Kumar, N. Moorthy Muthukrishnan, Abhishek Mahajan, P. Priyanka, G. Padmavathi, M. Nethra, R. Sneha, Meenakshi H Thakur. (2016). Statistical Parameter-based Automatic Liver Tumour Segmentation from Abdominal CT Scans: A Potential Radiomic Signature. 6th International Conference On Advances in Computing \& Communications, (ICACC). Procedia Computer Science 93 (2016). pp 446 - 452.

[50] Anuradha1 and Bhupinder Kaur. (2017). Infected Fruit Part Detection Using Different Segmentation Techniques. International Journals of control theory and applications. VOL 10. NO.10. pp 19-23.

[51] Jung-Shiong Chang, Hong-Yuan Mark Liaob, Maw-Kae Herb, Jun-Wei Hsieh. (1997). New automatic multi-level thresholding technique for segmentation of thermal images. Image and Vision Computing. VOL 15. PP 23 34.

[52] Yuan Been Chen. (2011). robust fully automatic scheme for general image segmentation. Digital Signal 
Processing. VOL 21. PP 87-99.

[53] L. P Clarke, R. P Velthijizen, M. A Camacho, J.J. Heine, M. Vaidya Nathan. MRI Segmentations: Methods and Applications. Magnetic Resonance Imaging. VOL 13, NO. 3, pp. 343-368.

[54] Gautam Pal, Suvojit Acharjee, Dwijen Rudrapaul, Dwijen Rudrapaul, Nilanjan Dey, (2015).Video segmentation using minimum ratio similarity measurement. Int. J. Image Mining, VOL 1, NO. 1. pp $87-110$.

[55] Deepa Parasar, Vijay R. Rathod. (2017).Particle swarm optimisation K-means clustering segmentation of foetus ultrasound image. Int. J. Signal and Imaging Systems
Engineering. VOL 10, NO. 1/2, pp 95-108.

[56] Yuri Boykon, Gareth Funka-Lea (2006),Graph Cuts and Efficient N-D Image Segmentation,International Journal of Computer Vision. VOL 70.NO. 2, pp 109-131.

[57] Fei Ni, Zhuang Fu, Qi Xin Cao, Yan Zheng Zhao. (2008). Image processing method for eyes location based on segmentation texture. Sensors and Actuators. pp 439451.

[58] Sourabh Srivastava, Satish Kumar Singh, Dhara Singh Hooda. (2015). Color sensing and image processingbased automatic soybean plant foliar disease severity detection and estimation. Multimedia Tools and Application, VOL 74. NO. 24. pp.11467-114 